\title{
Influence of Lactobacillus plantarum on yogurt fermentation properties and subsequent changes during postfermentation storage
}

\author{
Changkun Li, Jihong Song, Lai-yu Kwok, Jicheng Wang, Yan Dong, Haijing Yu, Qiangchuan Hou, \\ Heping Zhang, and Yongfu Chen ${ }^{1}$ \\ Key Laboratory of Dairy Biotechnology and Engineering, Ministry of Education P.R.C. Inner Mongolia Agricultural University, \\ Synergetic Innovation Center of Food Safety and Nutrition, Huhhot 010018, P. R. China
}

\begin{abstract}
This study aimed to evaluate the influence of $9 \mathrm{Lac}$ tobacillus plantarum with broad-spectrum antibacterial activity on fermented milk, including changes to the fermentation characteristics ( $\mathrm{pH}$, titration acidity, and viable counts), texture profile, relative content of volatile compounds, and sensory evaluation during 28-d storage at $4^{\circ} \mathrm{C}$. First, L. plantarum IMAU80106, IMAU10216, and IMAU70095 were selected as candidates for further study because of their excellent coagulation and proteolytic activities. Subsequently, these L. plantarum strains were supplemented to fermented milk produced by commercial yogurt starters (Streptococcus thermophilus and Lactobacillus delbrueckii ssp. bulgaricus) and a panel of parameters reflecting product quality was subsequently monitored during $28 \mathrm{~d}$ of postfermentation storage. The $\mathrm{pH}$ value and titration acidity of the fermented milk mildly fluctuated, whereas the L. plantarum viable counts remained stable along the storage period. Fourteen key volatile compounds were detected in the fermented milk by gas chromatography-mass spectrometry, and some flavor compounds were uniquely present in the L. plantarum-supplemented fermented milk (including 2,3-pentanedione, acetaldehyde, and acetate). No significant difference was shown in the sensory evaluation scores between samples with or without $L$. plantarum supplementation, but a gradual decrease was observed over storage in all samples. However, when $L$. plantarum was added, apparent shifts were observed in the overall quality of the fermented milk based on principal component analysis and multivariate ANOVA, particularly in the texture (adhesiveness) and volatile flavor compound profiles (acetaldehyde). Compared with L. plantarum IMAU80106 and IMAU10216, both the texture and volatile flavor profiles of IMAU70095 were closest to those of the control without adding the
\end{abstract}

Received August 11, 2016.

Accepted December 20, 2016.

${ }^{1}$ Corresponding author: nmgyfchen@126.com adjunct bacteria, suggesting that IMAU70095 might be the most suitable strain for further application in functional dairy product development. The current work has explored the potential of applying L. plantarum in fermented milk by performing thorough physical and chemical characterization. Our work is of intense interest to the dairy industry.

Key words: Lactobacillus plantarum, fermented milk, adjunct culture, postfermentation storage

\section{INTRODUCTION}

Fermented milk is produced by the acidification action of lactic acid bacteria (LAB) during metabolism, which results in important physicochemical, sensory, and microbiological changes in the milk (Casarotti et al., 2014). The primary LAB involved in food fermentation include the starter cultures, adjunct cultures, and probiotic cultures. In industrial production, commercial yogurt starters, namely Streptococcus thermophilus and Lactobacillus delbrueckii ssp. bulgaricus, are often used to ferment pasteurized milk (Fisberg and Machado, 2015); thus, dairy products are considered as the most suitable carriers for probiotics (Lourens-Hattingh and Viljoen, 2001). Probiotics are defined as "live microorganisms which when administered in adequate amounts confer a health benefit on the host" (FAO/WHO, 2001). Therefore, the development of novel functional products by incorporating probiotic bacteria in fermented milk is a current focus in the dairy industry (Georgieva et al., 2009). Adjunct cultures may also confer additional properties to the fermented products. For example, they may enhance the flavor, texture, or visual appearance of the products. The application of probiotic adjunct culture has also become a popular practice.

To provide health benefits, the probiotic adjunct culture must overcome the physical and chemical barriers such as acid and bile stress in the gastrointestinal tract (Del Piano et al., 2006) and maintain a high viability (at least $10^{6} \mathrm{cfu} / \mathrm{g}$ ) throughout the specified product shelf life (Tripathi and Giri, 2014). The most com- 
monly used probiotic bacteria are LAB; in particular, lactobacilli and bifidobacteria are considered to have the highest potential. Among the lactobacilli, L. plantarum is a versatile and widely distributed species. Its dual role as an indigenous human gut inhabitant and a safe starter culture in food fermentation (De Vries et al., 2006) has made it a preferred choice to be used in novel functional product development. Many previous reports found that consuming L. plantarum-containing fermented milk brings in vivo functionality to the host (Chiu et al., 2006; Nasrabadi et al., 2011). In our previous study, $9 \mathrm{~L}$. plantarum were identified to suppress the growth of 5 common foodborne pathogens (namely Escherichia coli, Staphylococcus aureus, Listeria monocytogenes, Salmonella typhimurium, and Shigella flexneri) that could be present in fermented milk (Li et al., 2015); thus, they have great potential to serve also as natural probiotic biopreservatives. Nevertheless, this continues to be a difficult task due to the stressful environment created for the probiotic bacteria during fermentation and storage.

On the other hand, a good adjunct bacterium should neither adversely affect product quality and sensory properties such as texture, taste, chemical content, and flavor nor enhance acidification during product storage. However, during the processes of milk fermentation and product storage, the viable bacteria including any adjunct culture would still continue to convert milk lactose to lactic acid and other flavor compounds (Kök-Tas et al., 2013), causing a series of physical and chemical changes. Although some previous studies have successfully selected adjunct cultures that do not alter the sensory profile and texture of prebiotic-containing yogurts (Hekmat and Reid, 2006; Kailasapathy, 2006), more comprehensive and objective evaluation of the changes occurred during fermented milk storage will be necessary to set up guidelines for product quality assurance.
The objective of this study was to test the effect of 9 L. plantarum strains on milk fermentation and postfermentation storage of the products over $28 \mathrm{~d}$. These L. plantarum strains were chosen based on their high in vitro tolerance to low $\mathrm{pH}$, artificial digestive juices, and bile salts, together with their strong anti-bacterial capacity in fermented milk (Li et al., 2015). To quantify the changes, we measured a wide spectrum of parameters, including the $\mathrm{pH}$, titratable acid (TA), viable counts, texture profile, sensory quality, and volatile flavor compounds, along the monitored time course. Data were then analyzed with principal component analysis (PCA) and multivariate ANOVA (MANOVA). Our long-term goal is to identify probiotic adjunct cultures that are suitable for developing novel functional fermented milk products.

\section{MATERIALS AND METHODS}

\section{Bacterial Isolates and Reagents}

Nine isolates of L. plantarum with broad inhibition activity against 5 enteric pathogens (Li et al., 2015) were obtained from the Lactic Acid Bacteria Culture Collection of the Key Laboratory of Dairy Biotechnology and Engineering, Inner Mongolia Agricultural University, Inner Mongolia, China. These bacteria were originally isolated from food and identified as L. plantarum using a combination of traditional physiological and biochemical identification methods and 16S rRNA gene sequence analysis; their $16 \mathrm{~S}$ rRNA gene sequences were submitted to the GenBank database (Table 1; Zhang et al., 2012). All isolates were stored long term in a skim milk medium (SMM, NZMP Co. Ltd., Wellington, New Zealand) at $-80^{\circ} \mathrm{C}$ and recovered in de Man, Rogosa, and Sharpe broth (Oxoid Ltd., Basingstoke, UK) at $37^{\circ} \mathrm{C}$ for $24 \mathrm{~h}$ following standard methods (Vinderola et al., 2000) before experiments. Commercial yogurt

Table 1. Origins of the 9 Lactobacillus plantarum strains

\begin{tabular}{lllll}
\hline Strain & $\begin{array}{l}\text { GenBank } \\
\text { accession no. }\end{array}$ & Sample type & Sampling region & Reference \\
\hline IMAU80106 & GU125529 & Pickle & Sichuan & Zhang et al., 2012 \\
IMAU10996 & HM218698 & Yogurt & Inner Mongolia & Zhang et al., 2012 \\
IMAU10216 & GU138544 & Sour dough & Inner Mongolia & Zhang et al., 2012 \\
IMAU70095 & GQ131211 & Sour porridge & Inner Mongolia & Bao et al., 2012 \\
IMAU70023 & GQ131139 & Sour porridge & Inner Mongolia & Yu et al., 2011 \\
IMAU30043 & FJ749637 & Koumiss & Xinjiang & Zhang et al., 2012 \\
IMAU50045 & FJ749445 & Dairy fan acid whey & Yunnan & Yu et al., 2012 \\
IMAU30162 & FJ749719 & Koumiss & Xinjiang & Zhang et al., 2012 \\
IMAU40082 & FJ749357 & Fermented yak milk & Qinghai & Bao et al., 2012 \\
\hline
\end{tabular}


starter cultures (YF-L904), which contained S. thermophilus and L. delbrueckii ssp. bulgaricus, were provided by Chr. Hansen Co. Ltd. (Hørsholm, Denmark) in direct vat set form.

\section{Milk-Coagulating Activity of L. plantarum}

The milk-coagulating activity of the $9 \mathrm{~L}$. plantarum strains was measured by a modified method based on Georgieva et al. (2009). Three skim milk-containing culture media were used: $10 \%$ reconstituted skim milks, $10 \%$ reconstituted skim milk supplemented with $1 \%$ glucose, and $1 \%$ yeast extract powder (Biosharp, Hefei, China). All 3 culture media were blended with water at $50^{\circ} \mathrm{C}$ and were brought to $121^{\circ} \mathrm{C}$ for $7 \mathrm{~min}$ (Wang et al., 2009). The $9 \mathrm{~L}$. plantarum isolates were washed with phosphate buffer, before being inoculated separately in the 3 types of skim milk-containing media at a concentration of $5 \times 10^{6} \mathrm{cfu} / \mathrm{mL}$. The inoculated cultures were incubated at $42^{\circ} \mathrm{C}$ for $16 \mathrm{~h}$ before recording their curdling status.

\section{Proteolytic Activity of L. plantarum}

Fermented milks were made in culture flasks by inoculating the $9 \mathrm{~L}$. plantarum strains each at a concentration of $5 \times 10^{6} \mathrm{cfu} / \mathrm{mL}$ with a noninoculated milk control. The inoculated milk cultures were incubated at $37^{\circ} \mathrm{C}$ for $48 \mathrm{~h}$. The quantity of free amino nitrogen (FAN) was monitored using the ortho-phthalaldehyde method along the fermentation process (Church et al., 1983).

\section{Fermented Milk Production}

The reconstituted milk was prepared by blending and homogenizing $11.5 \%$ (wt/wt) whole milk powder (purchased from NZMP Co. Ltd.; contained $39.1 \mathrm{~g} / 100$ $\mathrm{g}$ of lactose, $26.8 \mathrm{~g} / 100 \mathrm{~g}$ of fat, and $25.0 \mathrm{~g} / 100 \mathrm{~g}$ of protein) and $6.5 \%$ sucrose with sterilized distilled water at $50^{\circ} \mathrm{C}$ at $20 \mathrm{MPa}$ with a high-pressure homogenizer (SRH, Shenlu, Shanghai). The milk mixture was then pasteurized at $95^{\circ} \mathrm{C}$ for 10 min. Fermented milks were made in culture flasks by inoculating $0.003 \%$ (wt/vol) seed cultures of a commercial yogurt starter YF-L904. Fermentation was carried out at $42^{\circ} \mathrm{C}$ until reaching $\mathrm{pH}$ 4.5. The resulting yogurt was immediately cooled to $4^{\circ} \mathrm{C}$ in an ice bath and inoculated with $L$. plantarum at a concentration of $5 \times 10^{6} \mathrm{cfu} / \mathrm{g}$. A control without L. plantarum was performed in parallel. The formation of coagulum was prevented by an additional agitation step carried out at $42^{\circ} \mathrm{C}$ for $10 \mathrm{~min}$. The final product was poured into sanitized screw-cap bottles and kept refrigerated at $4^{\circ} \mathrm{C}$ until the experiment ended. Samples were taken at $1,7,14,21$, and $28 \mathrm{~d}$ for further analysis. All attributes were measured in triplicate.

\section{Measurement of $\mathrm{pH}$ and TA}

The $\mathrm{pH}$ was measured at $20^{\circ} \mathrm{C}$ using a pHSJ-3F pH meter (Leici, Shanghai, China). Titratable acid content was determined by titration with $0.1 \mathrm{~N} \mathrm{NaOH}$ using phenolphthalein as the indicator (National Standards of the People's Republic of China 1996).

\section{Plate Count}

After evenly mixing the fermented milk, $1 \mathrm{~mL}$ of each sample was diluted with $9 \mathrm{~mL}$ of sterile $0.1 \%$ (wt/vol) peptone water (Oxoid Ltd.) and serially diluted. Viable bacterial counts of $L$. plantarum in the fermented milk were determined after incubating at $37^{\circ} \mathrm{C}$ for $48 \mathrm{~h}$ on de Man, Rogosa, Sharpe agar containing $10 \mathrm{mg} / \mathrm{L}$ of vancomycin (Oxoid Ltd.), and identification of $L$. plantarum was based on colony morphology.

\section{Texture Profile Analysis}

A modified method based on Damin et al. (2008) was used for texture profile analysis. Mainly, texture profile analysis data (including the full texture curve, hardness, cohesiveness, adhesiveness, springiness, resilience, gumminess and chewiness of the fermented milk) were collected and analyzed by the TA-XT Plus texture analyzer (Stable Micro Systems, Godalming, UK) with the flat bottom cylindrical probe type of SMS/40 (40 $\mathrm{mm}$ diameter). The viscosity was measured using a viscometer (DVE, Brookfield, Middleboro, MA).

Fermented milk syneresis was measured based on Aryana (2003). For each test, 3 cups of $20 \mathrm{~g}$ of fermented milk were inverted and poured onto the quantitative filter paper placed on top of a funnel. The quantity of filtrate collected after $1.5 \mathrm{~h}$ of drainage at $21^{\circ} \mathrm{C}$ was used as an index of the water-holding capacity of the fermented milk. The level of syneresis (\%) was calculated according to the following equation:

$$
\text { syneresis }(\%)=\frac{\text { filtrate collected }(\mathrm{g})}{20 \text { - } \mathrm{g} \text { sample }} \times 100 \% \text {. }
$$

\section{Determination of Volatile Flavor Compounds}

The volatile flavor compounds were extracted by solid-phase microextraction technique, according to Lee et al. (2003). Briefly, samples were magnetically stirred and homogenized in a flavor isolation apparatus for $20 \mathrm{~min}$ at $50^{\circ} \mathrm{C}$. The carboxen/polydimethylsiloxane 
$\mathrm{d}_{\mathrm{f}}$ (thickness of the deep filtration layer) $75-\mu \mathrm{m}$ fiber (Supelco, Bellefonte, PA) trapped the headspace volatile compounds for $30 \mathrm{~min}$ at $40^{\circ} \mathrm{C}$ during stirring. The volatile compounds isolated by carboxen/polydimethylsiloxane were desorbed in the injector port of a GC-MS (7697-GC-7980-MS-5977, Agilent Technologies, Santa Clara, CA). Volatile flavor compounds were measured by GC-MS with the use of a capillary column (HP Innowax; $30 \mathrm{~m} \times 0.25 \mathrm{~mm}$; ID $0.25 \mu \mathrm{m}$ film thickness; Agilent Technologies). The carrier gas was helium with a flow of $1.2 \mathrm{~mL} / \mathrm{min}$ in splitless mode. The temperature of the GC-MS injection port was $250^{\circ} \mathrm{C}$. The temperature program was isothermal at $45^{\circ} \mathrm{C}$ for $5 \mathrm{~min}$, followed by an elevation of $4^{\circ} \mathrm{C} / \mathrm{min}$ until $220^{\circ} \mathrm{C}$ for 5 min. The MS was operated in the electron impact mode with an electron impact energy of $70 \mathrm{eV}$ and ion source temperature of $200^{\circ} \mathrm{C}$. Data were collected at a rate of $0.7 \mathrm{scan} / \mathrm{s}$ over a range of $\mathrm{m} / \mathrm{z} 40$ to 650 . Compounds were identified by comparing (1) the retention index with those described in literature and the mass spectra in the NIST11 (https://www.nist.gov/srd/ nist-standard-reference-database-1a-v14) and (2) GC retention indices of C3-C25 n-alkanes (AccuStandard Inc., New Haven, CT) detected under the same chromatographic conditions. Furthermore, acetoin, acetaldehyde, and acetate were used as standards to confirm the identifications. Acetoin, acetaldehyde, and acetate were obtained from Sigma-Aldrich (St. Louis, MO). All relative contents of compounds were expressed as percentages of their peak areas on the cyclohexanone $(5 \mu \mathrm{g} / \mathrm{mL})$ peak area.

\section{Sensory Evaluation}

Thirty panelists were selected and invited to take part in the sensory evaluation. Samples $(30 \mathrm{~mL})$ were served in polystyrene cups coded with random digit numbers and were presented in individual booths illuminated with white light under controlled humidity and temperature $\left(25^{\circ} \mathrm{C}\right)$. Participants were instructed to eat a cream cracker and drink water between samples to cleanse the palate. Sample acceptance was rated using a 10-point hybrid hedonic scale, where $1=$ disliked extremely and $10=$ liked extremely. The sensory attributes, namely flavor, texture, and appearance, were also evaluated. Overall mean score was calculated for each sample by averaging the score of every participant.

\section{Statistical Analysis}

All experiments were performed twice and in triplicate. Data are expressed as means \pm SD. Differences between groups were analyzed by ANOVA using SPSS Base 19.0 (IBM Ltd., New York, NY). The overall dif- ference between sample groups were assessed with PCA (performed with R software; https://www.r-project. org/) and MANOVA (performed with Matlab 2011b, MathWorks Inc., Natick, MA). Other figures were created by the OriginPro 2015 software (OriginLab Co., Northampton, MA).

\section{RESULTS}

\section{Coagulation Ability and Proteolytic Activity}

All 9 L. plantarum were analyzed for their ability to coagulate skim milk and skim milk supplemented with an additional nitrogen or carbon source. All the strains were only able to coagulate the yeast extract containing skim milk, with a shorter coagulation time for strains IMAU10216, IMAU80106, and IMAU70095 $(8.0,8.5$, and $9.3 \mathrm{~h}$, respectively; other strains required over $10 \mathrm{~h}$ ).

The proteolytic ability of all the $9 \mathrm{~L}$. plantarum were assessed by monitoring the release of fermentative FAN (data not shown). Data of the 3 strongest proteolytic strains (IMAU80106, IMAU10216, and IMAU70095) are shown in Figure 1. Because these strains (IMAU80106, IMAU10216, and IMAU70095) showed the highest proteolytic and coagulation abilities, most of the subsequent analyses were only performed on them.

\section{Titratable Acid and $\mathrm{pH}$}

Titratable acidity and $\mathrm{pH}$ are key indicators for assessing the acidity of fermented milk. The $\mathrm{pH}$ of the fermented milk dropped during the first $14 \mathrm{~d}$ of storage, followed by a stable period between d 14 and 21 . The fermented milk $\mathrm{pH}$ values significantly $(P<$ 0.05 for all groups) increased at the last week (Figure 2a). A similar pattern of changes in TA was observed in IMAU10216-containing fermented milk and the control without L. plantarum. The IMAU70095- and IMAU80106-containing fermented milk had a generally higher and lower TA level, respectively, over the monitored storage period (Figure $2 \mathrm{~b}$ ).

\section{Viable Counts of L. plantarum}

The viable count of all the L. plantarum-containing fermented milk was stable over the 28-d storage period (data not shown). The viable counts of fermented milk containing the strains IMAU80106, IMAU10216, and IMAU70095 (strongest milk coagulating and proteolytic strains) are shown in Supplemental Table S1 (https:// doi.org/10.3168/jds.2016-11864). No significant difference was observed at the start and the end of the storage period $(P>0.05)$. 


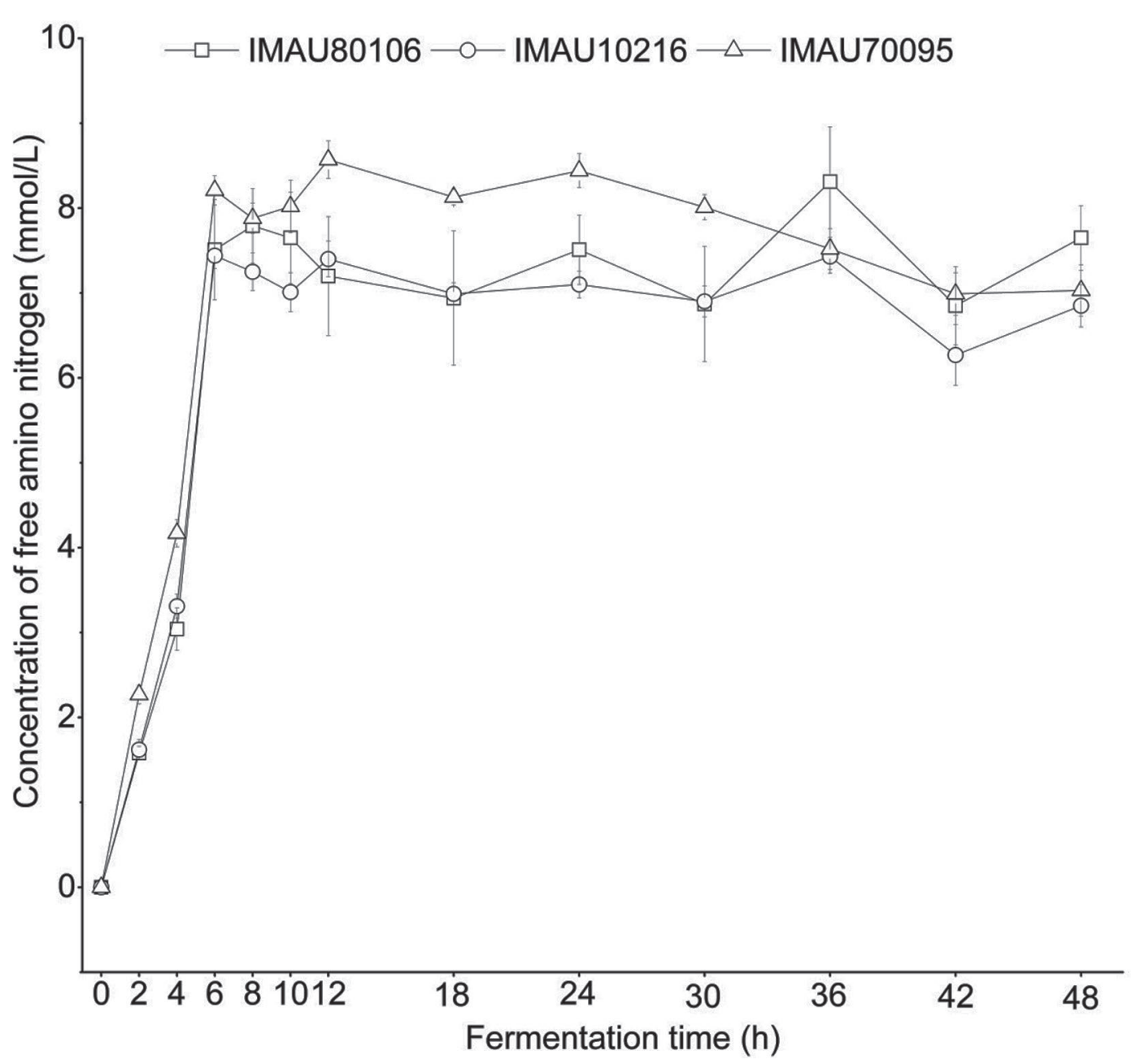

Figure 1. Change in free amino nitrogen concentration during Lactobacillus plantarum single milk fermentation. Error bars represent SD.

\section{Texture Profile Analysis}

To evaluate the physical characteristics of the fermented milk, 9 texture parameters (hardness, syneresis, cohesiveness, adhesiveness, springiness, gumminess, chewiness, resilience, and viscosity) were measured and compared along the postfermentation storage (Table 2). To better understand the effect of $L$. plantarum and the storage time on the texture profile of fermented milk samples, PCA and MANOVA analyses were performed (Figure 3). Symbols representing the texture profile of IMAU80106-containing fermented milk separated moderately from those of the IMAU10216, IMAU70095, and the control on the PCA score plot grouped based on the L. plantarum used (Figure 3a); the significant difference between the IMAU80106 from other groups was confirmed by MANOVA ( $P=0.046$; Figure $3 \mathrm{~b})$. The factor loadings also revealed strong positive correlations between the texture of IMAU80106 samples with resilience (factor score $=0.83$ ) and adhesiveness (0.82), which contributed most to the distinction between the IMAU80106-supplemented fermented milk from other groups (Figure 3a). Higher levels in both the adhesiveness (ranging from -69.81 to $-80.79 \mathrm{~g} \cdot \mathrm{s}$ for IMAU80106 group versus -77.73 to $-116.76 \mathrm{~g} \cdot \mathrm{s}$ for other groups) and resilience (ranging from 0.04 to 0.11 for IMAU80106 group versus 0.02 to 0.05 for other groups) were observed in the IMAU80106-supplemented samples (Table 2). The adhesiveness of fermented milk samples showed a decreasing trend along the storage period; however, the reduction in adhesiveness of fermented milks supplemented with IMAU80106 was milder compared with that of the other groups (Table 2).

When the texture profile of fermented milk samples were grouped based on storage time by PCA (Figure 3c), significant differences were observed between sample groups of all time points $(P<0.05)$ except for 
d 21 and 28 as supported by the results of MANOVA (Figure 3d). The attributes of hardness (0.98), gumminess (0.96), and chewiness (0.78) were positively correlated with principal component $(\mathbf{P C})$ 1, suggesting that they were the leading factors in distinguishing the textural profile of samples at d 21 and 28 from the other groups. Furthermore, no significant changes were detected in the hardness and gumminess of $L$. plantarum-containing samples throughout the storage period $(P>0.05$; Table 2$)$.

\section{Analysis of Volatile Flavor Compound Profile}

A total of 14 major volatile flavor compounds were detected in the fermented milk samples (Table 3), and their identified detail information shown in Supplemental Table S2 (https://doi.org/10.3168/jds.2016-11864). On the PCA score plot grouped based on L. plantarum strains, distinct groupings could be observed (with PC1 and PC2 explained for 31.44 and $20.57 \%$ of variance, respectively; Figure 4a); the volatile flavor compound profiles of IMAU10216- and IMAU80160-supplemented fermented milks were different from one another $(P<$ $0.05)$, as well as from the control and IMAU70095 groups $(P<0.05)$ as determined by MANOVA (Figure $4 \mathrm{~b})$. The volatile flavor compounds, 2-nonanone (0.84), heptanal (0.86), and 2-heptanone (0.71), contributed most to $\mathrm{PC} 1$, whereas acetate $(0.60)$ and n-heptanol (0.66) strongly and negatively contributed to PC2 (Figures 4a and $4 \mathrm{c}$ ). Apparent overlapping of symbols representing the volatile flavor compound profiles at different time points when the results of PCA were grouped based on storage time (Figure 4c). Nevertheless, the results of MANOVA indicated that only the 7- and 14-d samples exhibited no significant difference $(P=0.53)$. For all the samples, the major volatile flavor compounds were n-heptanol, 2-heptanone, 1-pentanol, and hexanol (ranging between 12.64-44.37, 12.44-31.04, 9.39-30.47, and 37.51-66.75, respectively) during the postfermentation storage. In addition, 3 of the flavor volatile compounds, 2,3-pentanedione (samples with IMAU80106 or IMAU70095), acetaldehyde (samples with IMAU80106 or IMAU10216), and acetate (samples with IMAU10216 or IMAU70095), were found to be uniquely present in L. plantarum-containing samples but not the control group with this bacterium (Table 3), suggesting that they were produced by the metabolism of $L$. plantarum.

\section{Sensory Evaluation}

Results of the sensory evaluation are presented in Supplemental Table S3 (https://doi.org/10.3168/ jds.2016-11864). The scores for flavor, texture, and appearance showed a mild decreasing trend for all the groups along the storage period. No significant difference was detected between the $L$. plantarum and the control groups in any of the measured sensory attributes along the $28 \mathrm{~d}$ of storage $(P>0.05)$.

\section{Overall Quality of Fermented Milk Produced With or Without L. plantarum}

To assess the overall quality of the fermented milk samples of all groups, PCA and MANOVA analyses were performed based on all the measured parameters, namely the profiles of texture and volatile flavor com-

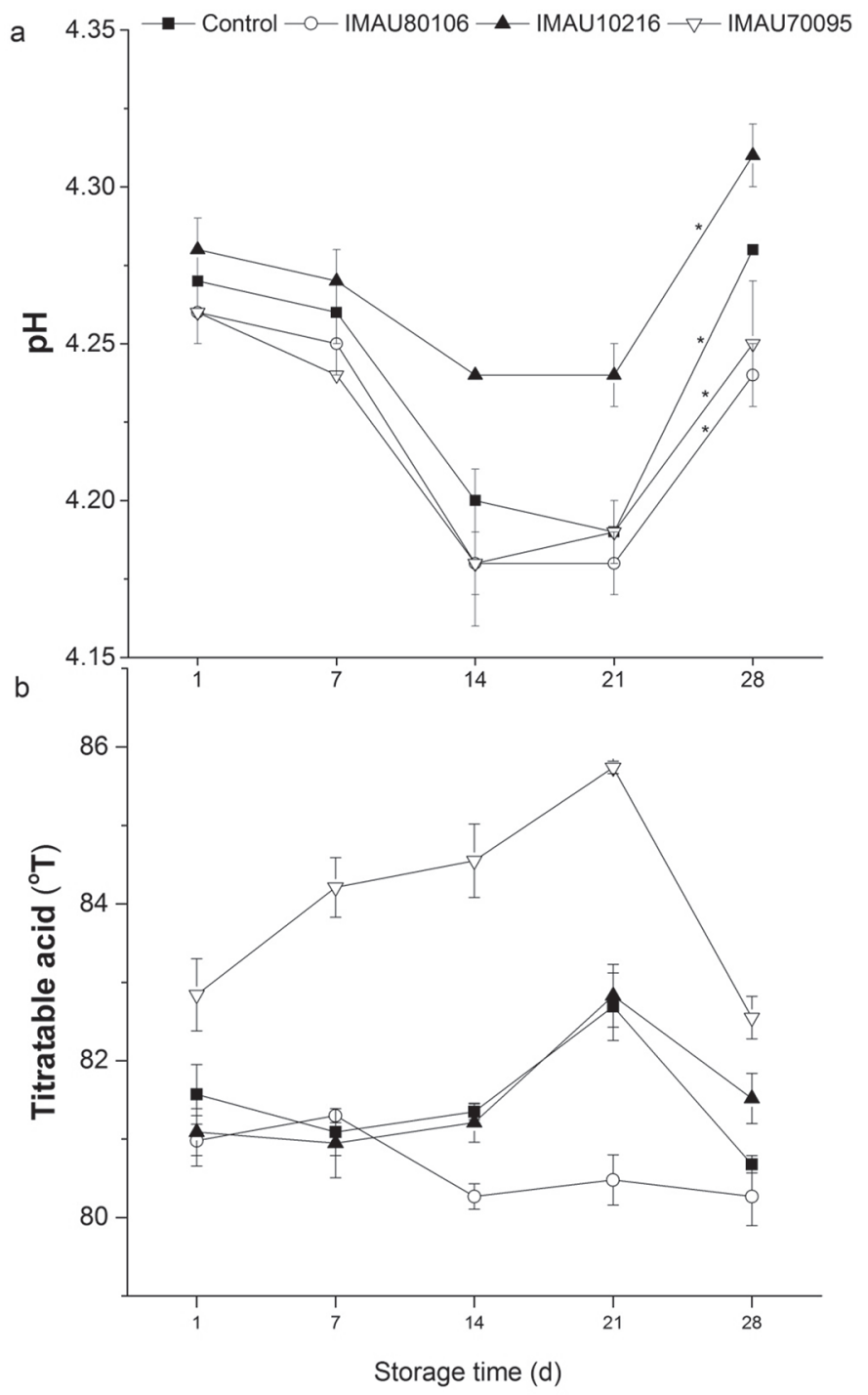

Figure 2. Effect of storage on (a) pH and (b) titratable acid concentration of fermented milk. Data from the fermented milk produced by the strains IMAU80106, IMAU10216, IMAU70095, and control (without $L$. plantarum) are represented by $\bigcirc, \boldsymbol{\Lambda}, \nabla$, and $\boldsymbol{\square}$, respectively. Error bars represent SD. $* P<0.05$. 


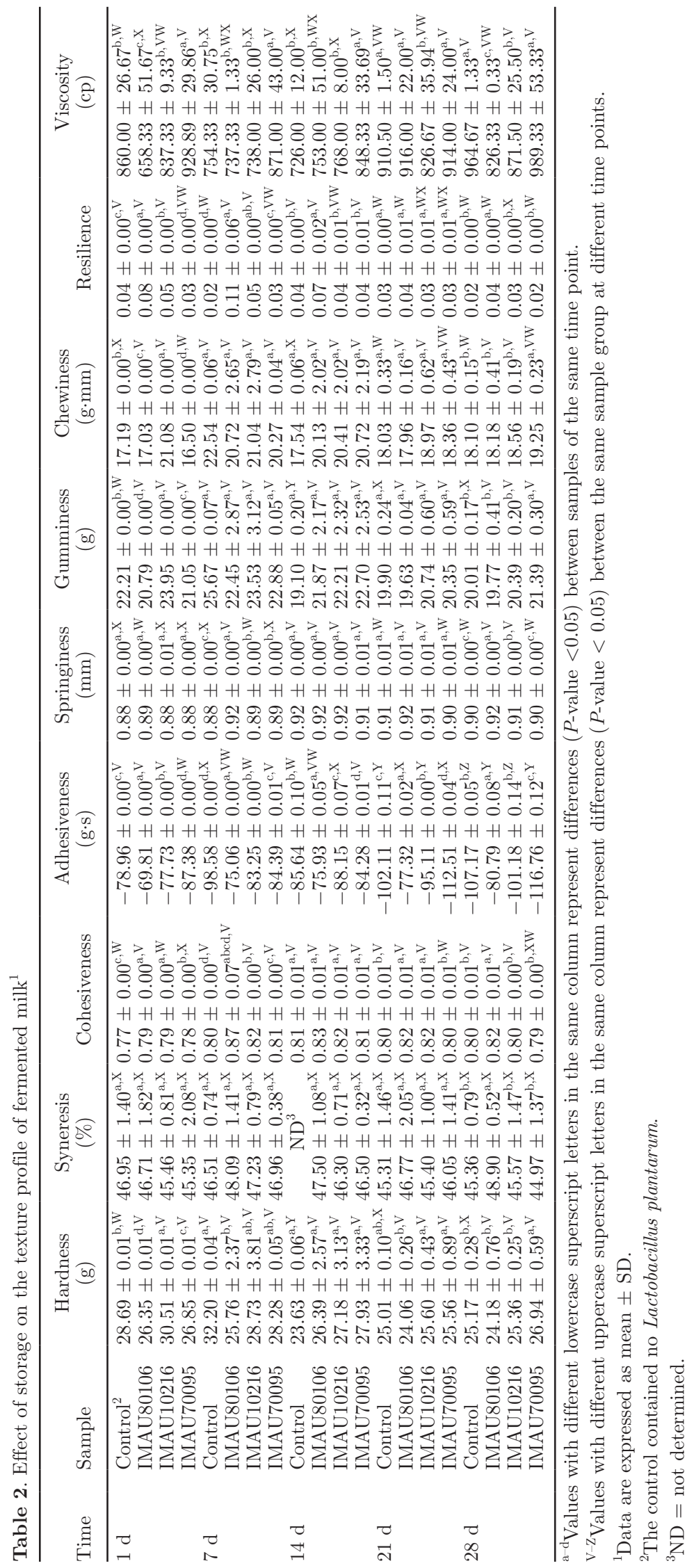


pounds together with the sensory attributes (flavor, texture, and appearance).

A clear clustering pattern was formed on the score plot when the samples were grouped based on the $L$. plantarum strains (Figure 5a), suggesting that adding L. plantarum into the fermented milk affected the fermented milk quality over the storage period; significant differences between the control and L. plantarumsupplemented groups were confirmed by MANOVA $(P$ $<0.05$; Figure 5b). In contrast, when the PCA results were grouped based on postfermentation storage time, no clear clustering pattern was identified (Figure 5c). Nevertheless, symbols representing samples of different storage time were found distributed from the right to the left side of the plot as the storage time increased, indicating gradual changes of the overall quality of the samples upon prolonged storage. This result was supported by MANOVA that revealed significant differences in the overall sample quality between samples of different storage time points $(P<0.05$ in all cases; Figure 5d). Moreover, the PCA results found 8 major determining factors contributing to $\mathrm{PC} 1$, which were the leading factors that distinguished samples stored for different time. The 4 factors, adhesiveness $(0.75)$, flavor (0.68), acetaldehyde (0.68), and texture (0.63), correlated with the early phase of sample storage, whereas the other 4 factors, viscosity $(-0.60)$, 2-nonanone $(-0.61), 2$-heptanone $(-0.63)$, and heptanal $(-0.72)$, correlated more to samples subjected to longer term storage, as symbols representing these groups (d 21 and 28) mostly had a negative PC1 value (Figure 5c).

\section{DISCUSSION}

The species L. plantarum has dual role as an indigenous human gut inhabitant and a safe starter culture in food fermentation; thus, it carries great potential to be used in functional dairy product development.
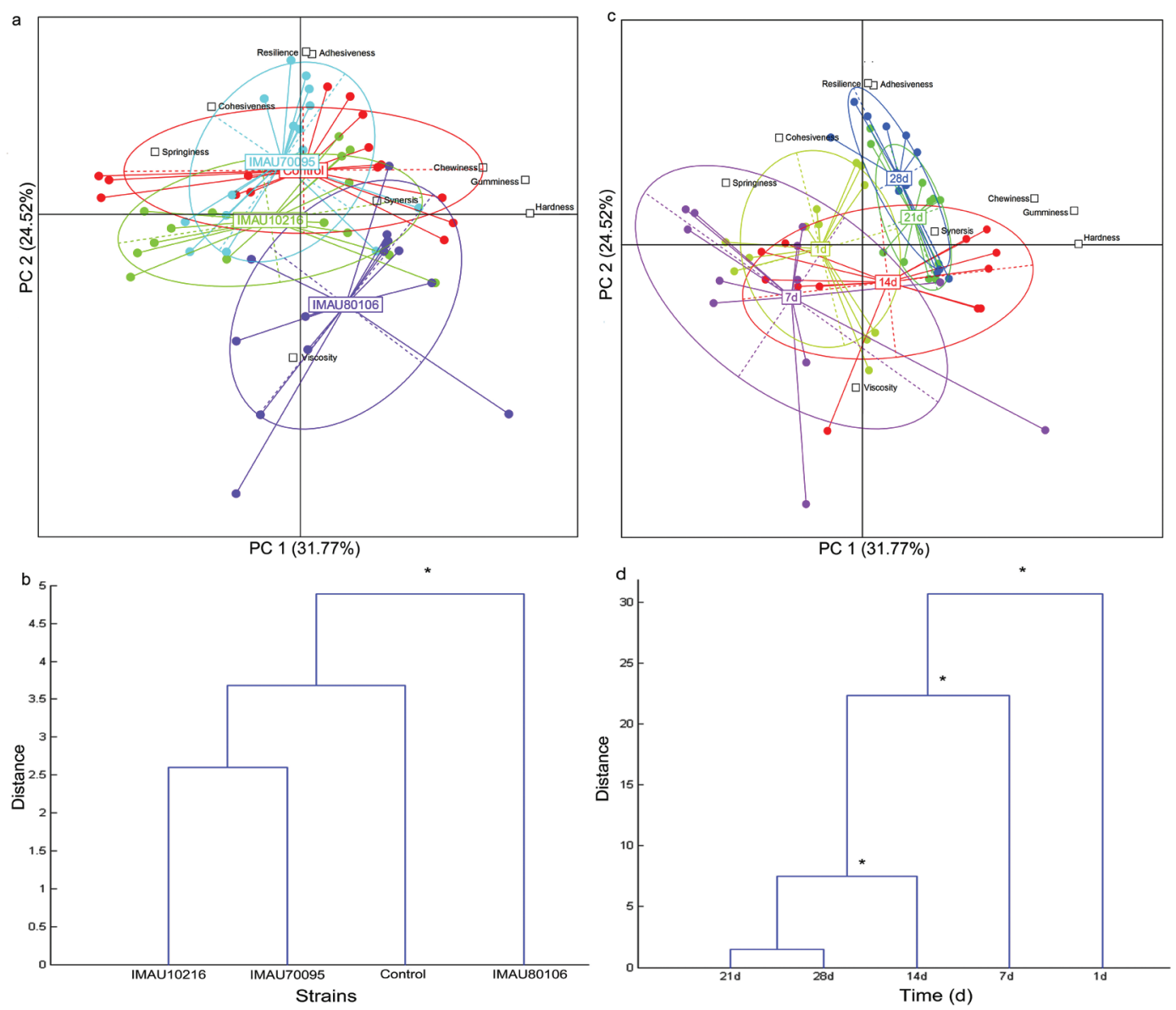

Figure 3. Principal component (PC) analysis and multivariate ANOVA based on fermented milk texture profile, grouped based on Lactobacillus plantarum strain used ( $\mathrm{a}$ and $\mathrm{b}$ ) and product postfermentation storage time (c and d). For PC analysis score and loading plots, each dot represents the data set of one sample. Samples of the same group (either representing used L. plantarum strain or postfermentation storage time) are represented by symbols of the same color on each plot. The control group contained no L. plantarum. ${ }^{*} P<0.05$. Color version available online. 


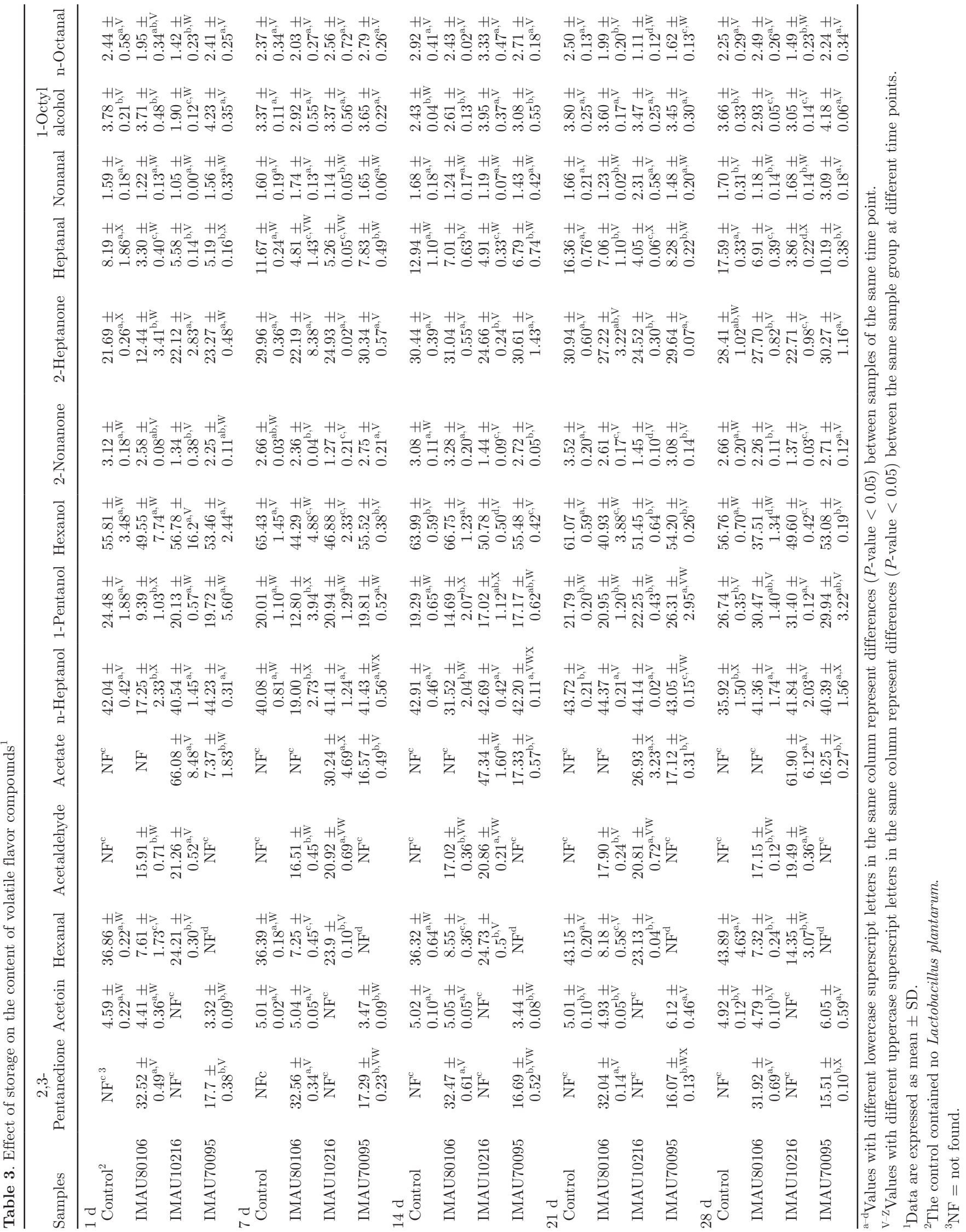


The most important factors to consider when probiotic adjunct cultures are to be applied in developing novel dairy products are its effect on the fermentation process, product quality, and the ultimate acceptability to consumers. Therefore, our study investigated whether and how the addition of different $L$. plantarum strains affected these aspects.

To assess the effect of adding L. plantarum on the milk fermentation, the proteolytic activity, coagulation ability, and release of FAN were compared between fermented milks made with and without L. plantarum. Only milk containing yeast extract were coagulated by the tested $L$. plantarum (particularly IMAU80106, IMAU10216, and IMAU70095, which had a coagulation time of less than $10 \mathrm{~h}$ ). Yeast extract acts as a source of nitrogen, vitamins, and growth factors, which might have accelerated the growth of these probiotic bacteria (Hekmat et al., 2009). Moreover, these 3 strains (IMAU80106, IMAU10216, and IMAU70095) released the highest level of FAN, which may indicate their intrinsically high proteolytic capacity. However, our data observed no direct correlation between the FAN content of fermented milk containing different $L$. plantarum strains and the studied texture and flavor parameters (unpublished data). In general, Lactobacillus uses casein by their cell-envelope proteinases that degrade the protein into oligopeptides, which are subsequently taken up by the cells via peptide transport systems. The metabolic capacities of the adjunct culture are also of industrial interest because their released metabolic end products (e.g., peptides, AA, and other derivatives) may influence the texture and flavor of the fermented milk products (Savijoki et al., 2006).

To investigate whether the addition of L. plantarum would change the fermented milk quality upon postfermentation storage, we monitored (for $28 \mathrm{~d}$ ) and
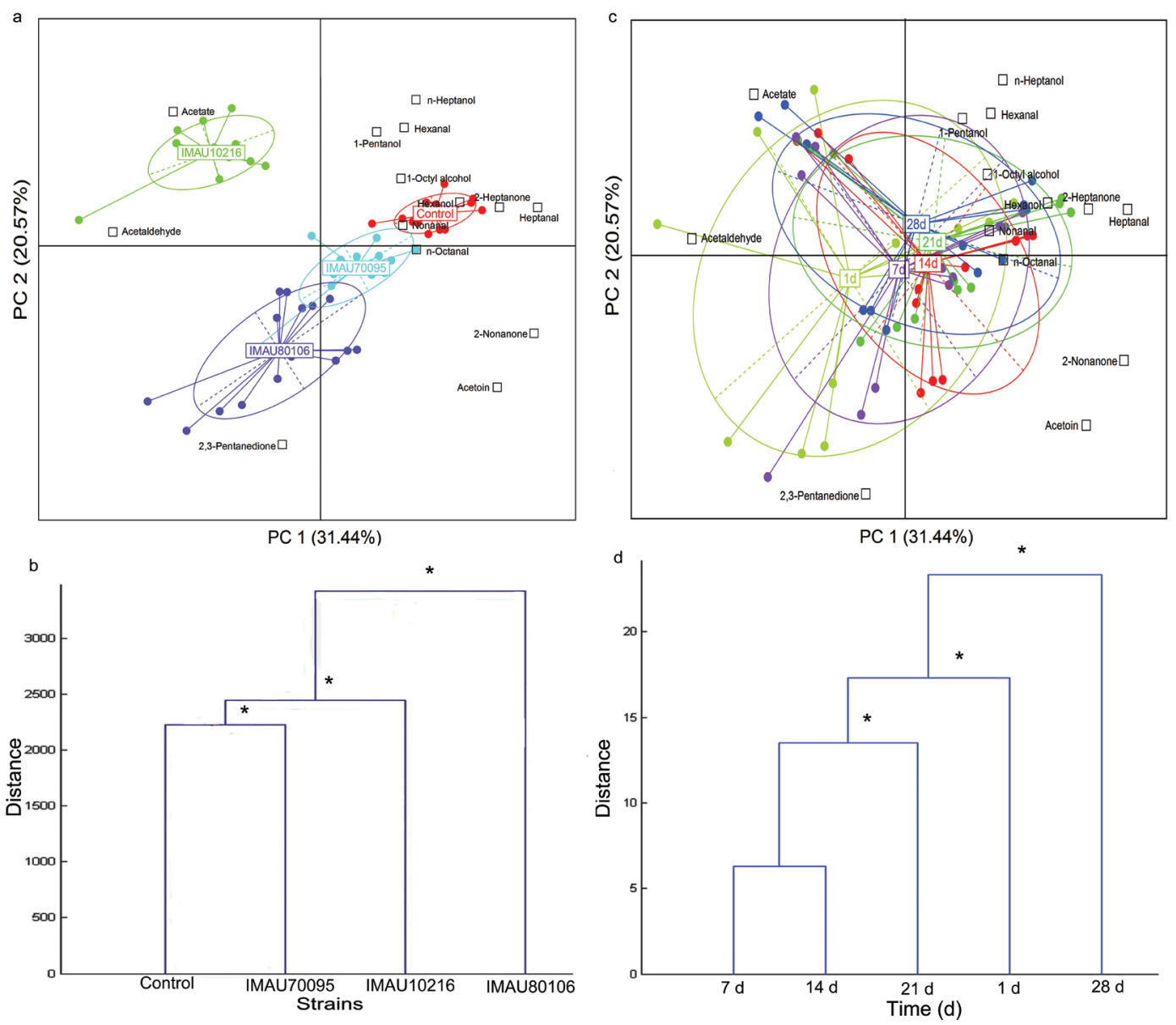

Figure 4. Principal component (PC) analysis and multivariate ANOVA based on fermented milk volatile flavor compound profile, grouped based on Lactobacillus plantarum strain used ( $\mathrm{a}$ and $\mathrm{b}$ ) and product postfermentation storage time (c and d). For PC analysis score and loading plots, each dot represents the data set of one sample. Samples of the same group (either representing used L. plantarum strain or postfermentation storage time) are represented by symbols of the same color on each plot. The control group contained no L. plantarum. ${ }^{*} P<0.05$. Color version available online. 

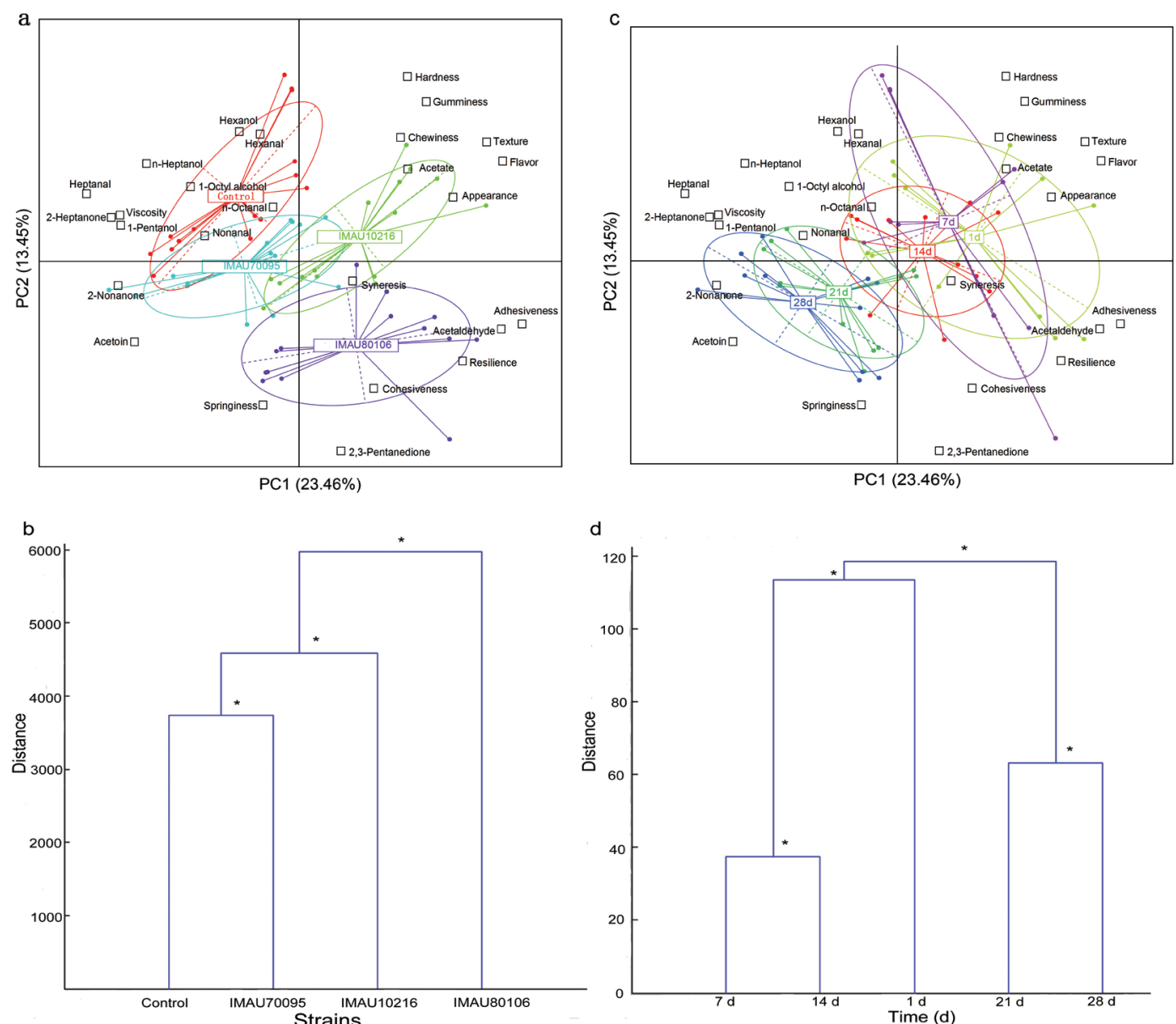

Figure 5. Principal component (PC) analysis and multivariate ANOVA based on fermented milk texture profile, volatile flavor compound profile, and sensory evaluation, grouped based on Lactobacillus plantarum strain used (a and b) and product postfermentation storage time (c and d). For PC analysis score and loading plots, each dot represents the data set of one sample. Samples of the same group (either representing used L. plantarum strain or postfermentation storage time) are represented by symbols of the same color on each plot. The control group contained no $L$. plantarum. ${ }^{*} P<0.05$. Color version available online.

compared several parameters of the yogurts produced with and without the addition of $L$. plantarum strains (only IMAU80106, IMAU10216, and IMAU70095 were studied, due to their high coagulative and proteolytic activities).

Among the various criteria that are taken into account for novel food product development, sensory quality (i.e., customer acceptability and preferences) should be of prime consideration (Hough et al., 2007; Allgeyer et al., 2010; Cheng, 2010). Sensory quality can be rated by the flavor, texture, and appearance (Hekmat and Reid, 2006), and no adverse effect on the taste or aroma of the products should be introduced when probiotics or adjunct cultures are to be applied (Heller, 2001). Our results show that the addition of any of the tested L. plantarum strains did not exert a negative effect on the product sensory quality.
The overall quality of all samples was evaluated by PCA and MANOVA (Figure 5). On the PCA score and loading plot, products fermented with the addition of the 3 L. plantarum were distributed to different quadrants (Figure 5a), suggesting that the choice of probiotic or adjunct strain used might exert specific effects on the final product quality. Symbols representing the quality profiles of IMAU70095-containing fermented milk sample were located closest to those of the controls, suggesting that their product quality deviated least from the control without adjunct strain. These results indicate that IMAU70095 might be the most suitable strain for further commercial application in fermented milk development.

By performing PCA based on all or some of the measured parameters, it is possible to relate the individual factors that have a key influence on product 
quality. On the PCA plot of the overall quality, the flavor (particularly acetaldehyde) and texture (especially adhesiveness) profiles were strongly correlated with PC1, whereas hardness and gumminess were correlated more with $\mathrm{PC} 2$; these results suggest that they were some of the key factors in distinguishing the different groups of samples. The volatile flavor compounds in fermented milk gave rise to the taste and aroma of the final products. Apparent shifts were observed in the volatile compound profiles when different $L$. plantarum strains were added to the fermented milk samples. By PCA and MANOVA, the volatile flavor compound profile of IMAU70095 strain was most similar to that of the control.

During the fermentation or postfermentation storage of yogurt, probiotics or adjunct cultures could cause many complex physical and chemical changes, such as proteolysis and acidification, which involves the gradual casein hydrolysis to form polypeptides, AA, and other compounds (Christensen et al., 1999; Shihata and Shah, 2000). Lactobacillus plantarum may contribute to the secondary proteolysis to release small peptides and free AA that act as major precursors of specific flavor compounds (Briggiler-Marcó et al., 2007). We detected the flavor compounds, 2,3-pentanedione, acetaldehyde, and acetate, in the L. plantarum-supplemented fermented milk (Table 3). 2,3-Pentanedione is produced from threonine metabolism and is responsible for eliciting the yogurt odor (Pourahmad and Assadi, 2005), whereas the maximal acetate is produced under aerobic conditions and glucose limitation (Mayo et al., 2010). The samples with L. plantarum IMAU10216 had significantly higher acetate concentration $(26.93 \pm 3.23$ to $66.08 \pm 8.48)$. Acetate $(0.60)$ is a factor that was positively correlated with the PC2 axis (Figure 4a), which might be a critical differential parameter that distinguished IMAU10216-containing samples from the other samples. Although acetate production can enhance shelf life and improve microbial safety of fermented dairy foods, an overly high concentration may result in a distinct and undesirable vinegary flavor (GallardoEscamilla et al., 2005). Similar, Settachaimongkon et al. (2014) found that acetaldehyde, acetoin, and acetate are major flavor compounds that distinguish fermented samples produced by mixed cultures of $S$. thermophilus and L. delbrueckii ssp. bulgaricus from the controls.

In yogurt, the compound acetaldehyde possesses a pleasant fruity aroma odor at dilute concentrations (Cheng, 2010) and is the most prominent flavoring compound in yogurt (Ott et al., 1999, 2000; GallardoEscamilla et al., 2005). As shown in the results (Table 3 ), a high content of acetaldehyde was found in samples with IMAU80106 and IMAU10216; this may be an important factor that distinguishes them from other samples.

Among the adjunct culture-containing fermented milks, only the IMAU70095-supplemented ones had an undetectable level of hexanal. This compound causes a bean flavor in milk (Farnworth et al., 2007). One possible explanation for the absence of such compound was its uniquely possession of hexanal degradation capacity; efficient utilization of hexanal in soy milk by LAB has previously been reported by Desai et al. (2002).

Texture is another crucial factor that affects the quality of yogurt, which is mainly determined by the milk processing mode, starter culture, and postfermentation temperature used. Our PCA and MANOVA results (Figure $3 \mathrm{~b}$ ) revealed that the IMAU70095-containing samples possessed the smallest within-clade distance from the control without $L$. plantarum $(P>0.05)$ compared with the other 2 tested strains. This result suggests that L. plantarum IMAU70095 had the minimum effect on the overall texture quality of fermented milk over a 28-d-storage at $4^{\circ} \mathrm{C}$. When the PCA was grouped based on storage time, an apparent trend of shifting was observed on the PCA score and loading plot, suggesting an overall texture change occurred along the storage period $(P<0.05$; Figure $3 \mathrm{c})$.

Yogurts with high adhesiveness could lead to a greater degree of stickiness in the mouth, which is a negative property relating to the consistency and texture (Kailasapathy, 2006). In contrast, a lower adhesiveness makes fermented milk more acceptable for the consumers (Tamime and Robinson, 1999). The fermented milk samples with IMAU80106 had highest adhesiveness (ranging from -80.79 to $-69.81 \mathrm{~g} \cdot \mathrm{s}$ ).

Hardness is the peak force measured during the first compression cycle of texture analysis. The control samples experienced a significant decrease in hardness $(P<0.05)$ upon storage. However, the hardness and gumminess of $L$. plantarum-containing samples were not significantly different along the storage period $(P$ $>0.05)$. Indeed, they became more stable as storage extended. The exopolysaccharides secreted by probiotic LAB might have helped strengthen the hardness of dairy products (Duboc and Mollet, 2001).

To exert beneficial health effects on the host, potential probiotic adjunct cultures must be able to survive the food fermentation process and survive throughout the product shelf life. Dairy products are often used as a probiotics carrier. Thus, we estimated also the viability of L. plantarum upon fermented milk storage. Our results confirmed that fermented milk is a suitable carrier growth culture for the tested $L$. plantarum, which fulfills the US Food and Drug Administration recommendation of viable probiotic counts for probiotics- 
based functional foods of at least $10^{6} \mathrm{cfu} / \mathrm{g}$ (Tripathi and Giri, 2014).

\section{CONCLUSIONS}

Our study investigated the effect of adding different L. plantarum strains on fermentation and $28 \mathrm{~d}$ of postfermentation storage of yogurts. We found that the tested strains, L. plantarum IMAU80106, IMAU10216, and IMAU70095, exhibited the highest coagulation ability and proteolytic activity. Generally, L. plantarum survived well in the fermented milk both during and postfermentation, suggesting that the milk medium is a suitable carrier for this species. The addition of $L$. plantarum did not cause any negative effect on the sensory quality of the fermented milk nor halt the natural postfermentation changes of the yogurt; however, with such treatment, apparent shifts were observed in the overall quality of the fermented milk based on PCA and MANOVA, in particular, in the profiles of texture (adhesiveness) and volatile flavor compounds (acetaldehyde). Comparing to L. plantarum IMAU80106 and IMAU10216, both the texture and volatile flavor profiles of IMAU70095 were closest to that of the control without adding adjunct culture, suggesting that the postfermentative changes due to adjunct culture addition were likely strain specific and that IMAU70095 might be the most suitable strain for further application in functional dairy product development.

\section{ACKNOWLEDGMENTS}

This research was supported by the China Agriculture Research System (grant no. CARS-37, Huhot, China), and the Special Fund for Agro-scientific Research in the Public Interest (grant no. 201203009, Huhot, China). We declare no conflicts of interest. This work is in compliance with ethics requirements; our work does not contain clinical studies or patient data.

\section{REFERENCES}

Allgeyer, L. C., M. J. Miller, and S. Y. Lee. 2010. Sensory and microbiological quality of yogurt drinks with prebiotics and probiotics. J. Dairy Sci. 93:4471-4479.

Aryana, K. J. 2003. Folic acid fortified fat-free plain set yoghurt. Int. Dairy Technol. 56:219-222.

Bao, Y., Y. Zhang, H. Li, Y. Liu, S. Wang, X. Dong, F. Su, G. Yao, T. Sun, and H. Zhang. 2012. In vitro screen of Lactobacillus plantarum as probiotic bacteria and their fermented characteristics in soymilk. Ann. Microbiol. 62:1311-1320.

Briggiler-Marcó, M., M. L. Capra, A. Quiberoni, G. Vinderola, J. A. Reinheimer, and E. Hynes. 2007. Nonstarter Lactobacillus strains as adjunct cultures for cheese making: In vitro characterization and performance in two model cheeses. J. Dairy Sci. 90:4532-4542.

Casarotti, S. N., D. A. Monteiro, M. M. S. Moretti, and A. L. B. Penna. 2014. Influence of the combination of probiotic cultures during fermentation and storage of fermented milk. Food Res. Int. 59:67-75.

Cheng, H. 2010. Volatile flavor compounds in yogurt: A review. Crit. Rev. Food Sci. Nutr. 50:938-950.

Chiu, C. H., T. Y. Lu, Y. Y. Tseng, and T. M. Pan. 2006. The effects of Lactobacillus-fermented milk on lipid metabolism in hamsters fed on high-cholesterol diet. Appl. Microbiol. Biotechnol. 71:238-245.

Christensen, J. E., E. G. Dudley, J. A. Pederson, and J. L. Steele. 1999. Peptidases and amino acid catabolism in lactic acid bacteria. Antonie van Leeuwenhoek 76:217-246.

Church, F. C., H. E. Swaisgood, D. H. Porter, and G. L. Catignani. 1983. Spectrophotometric assay using o-phthaldialdehyde for determination of proteolysis in milk and isolated milk proteins. J. Dairy Sci. 66:1219-1227.

Damin, M. R., E. Minowa, M. R. Alcantara, and M. N. Oliveira. 2008. Effect of cold storage on culture viability and some rheological properties of fermented milk prepared with yoghurt and probiotic bacteria. J. Texture Stud. 39:40-55.

Del Piano, M. D., L. Morellic, G. P. Strozzib, S. Allesina, M. Barbab, F. Deidda, P. Lorenzini, M. Ballaré, F. Montino, M. Orsello, M. Sartori, E. Garello, S. Carmagnola, M. Pagliarulo, and L. Capurso. 2006. Probiotics: From research to consumer. Dig. Liver Dis. 38:S248-S255.

De Vries, M. C., E. E. Vaughan, M. Kleerebezem, and W. M. de Vos. 2006. Lactobacillus plantarum - Survival, functional and potential probiotic properties in the human intestinal tract. Int. Dairy J. 16:1018-1028.

Desai, A., D. Small, A. E. J. Mcgill, and N. P. Shah. 2002. Metabolism of raffinose and stachyose in reconstituted skim milk and of n-hexanal and pentanal in soymilk by bifidobacteria. Biosci. Microflora 21:245-250.

Duboc, P., and B. Mollet. 2001. Applications of exopolysaccharides in the dairy industry. Int. Dairy J. 11:759-768.

FAO/WHO (Food, Agriculture Organization/World Health Organization). 2001. Health and nutritional properties of probiotics in food including powder milk with live lactic acid bacteria. Report of a joint FAO/WHO expert consultation on evaluation of health and nutritional properties in food including powder milk with live lactic acid bacteria. Cordoba, Argentina.

Farnworth, E. R., I. Mainville, M. P. Desjardins, N. Gardner, I. Fliss, and C. Champagne. 2007. Growth of probiotic bacteria and bifidobacteria in a soy yogurt formulation. Int. J. Food Microbiol. 116:174-181.

Fisberg, M., and R. Machado. 2015. History of yogurt and current patterns of consumption. Nutr. Rev. 73(Suppl. 1):4-7.

Gallardo-Escamilla, F. J., A. L. Kelly, and C. M. Delahunty. 2005. Influence of starter culture on flavor and headspace volatile profiles of fermented whey and whey produced from fermented milk. J. Dairy Sci. 88:3745-3753.

Georgieva, R., I. Iliev, T. Haertlé, J.-M. Chobert, I. Ivanova, and S. Danova. 2009. Technological properties of candidate probiotic Lactobacillus plantarum strains. Int. Dairy J. 19:696-702.

Hekmat, S., and G. Reid. 2006. Sensory properties of probiotic yogurt is comparable to standard yogurt. Nutr. Res. Rev. 26:163-166.

Hekmat, S., H. Soltani, and G. Reid. 2009. Growth and survival of Lactobacillus reuteri RC-14 and Lactobacillus rhamnosus GR-1 in yogurt for use as a functional food. Innov. Food Sci. Emerg. Technol. 10:293-296.

Heller, K. J. 2001. Probiotic bacteria in fermented foods: Product characteristics and starter organisms. Am. J. Clin. Nutr. 73:374S379 S.

Hough, G., M. L. Calle, C. Serrat, and A. Curia. 2007. Number of consumers necessary for shelf life estimations based on survival analysis statistics. Food Qual. Prefer. 18:771-775.

Kök-Tas, T., A. C. Seydim, B. Özer, and Z. B. Guzel-Seydim. 2013. Effects of different fermentation parameters on quality characteristics of kefir. J. Dairy Sci. 96:780-789.

Kailasapathy, K. 2006. Survival of free and encapsulated probiotic bacteria and their effect on the sensory properties of yogurt. LWT Food Sci. Technol. (Campinas) 39:1221-1227. 
Lee, J. H., R. Diono, G. Kim, and D. B. Min. 2003. Optimization of solid phase microextraction analysis for the headspace volatile compounds of parmesan cheese. J. Agric. Food Chem. 51:11361140 .

Li, C., Y. Chen, L. Y. Kwok, X. Chen, H. Yu, H. Yang, J. Yang, J. Xue, T. Sun, and H. Zhang. 2015. Identification of potential probiotic Lactobacillus plantarum isolates with broad-spectrum antibacterial activity. Dairy Sci. Technol. 95:381-392.

Lourens-Hattingh, A., and B. C. Viljoen. 2001. Review: Yoghurt as probiotic carrier in food. Int. Dairy J. 11:1-17.

Mayo, B., T. Aleksandrzak-Piekarczyk, M. Fernández, M. Kowalczyk, P. Álvarez-Martín, and J. Bardowsk. 2010. Updates in the metabolism of lactic acid bacteria. Biotechnology of Lactic Acid Bacteria Novel Applications. Wiley-Blackwell, Oxford, UK.

Nasrabadi, M. H., H. Aboutalebi, M. T. Ebrahimi, and F. Zahedi. 2011. The healing effect of Lactobacillus plantarum isolated from Iranian traditional cheese on gastric ulcer in rats. Afr. J. Pharm. Pharmacol. 5:1446-1451.

National Standards of the People's Republic of China. 1996. Method for analysis of hygienic standard of milk and milk products. Bulletin 5009:46 (CNS, Beijing, China.).

Ott, A., J. E. Germond, M. Baumgartner, and A. Chaintreau. 1999 Aroma comparisons of traditional and mild yogurts: Headspace gas chromatography quantification of volatiles and origin of adiketons. J. Agric. Food Chem. 47:2379-2385.

Ott, A., J. E. Germond, and A. Chaintreau. 2000. Vicinal diketon formation in yogurt: Precursors and effect of branched chain amino acids. J. Agric. Food Chem. 48:724-731.

Pourahmad, R., and M. M. Assadi. 2005. Yoghurt production by Iranian native starter cultures. Nutr. Food Sci. 35:410-415.

Savijoki, K., H. Ingmer, and P. Varmanen. 2006. Proteolytic systems of lactic acid bacteria. Appl. Microbiol. Biotechnol. 71:394-406.
Settachaimongkon, S., M. J. Robert Nout, E. C. Antunes Fernandes, K. A. Hettinga, J. M. Vervoort, T. C. M. van Hooijdonk, M. H. Zwietering, E. J. Smid, and H. J. F. van Valenberg. 2014. Influence of different proteolytic strains of Streptococcus thermophilus in coculture with Lactobacillus delbrueckii ssp. bulgaricus on the metabolite profile of set-yoghurt. Int. J. Food Microbiol. 177:29-36.

Shihata, A., and N. P. Shah. 2000. Proteolytic profile of yoghurt and probiotic bacteria. Int. Dairy J. 10:401-408.

Tamime, A. Y., and R. K. Robinson. 1999. Yoghurt Science and Technology. 2nd ed. CRC Press. New York.

Tripathi, M. K., and S. K. Giri. 2014. Probiotic functional foods: Survival of probiotics during processing and storage. J. Funct. Foods 9:225-241.

Vinderola, C. G., N. Bailo, and J. A. Reinheimer. 2000. Survival of probiotic microflora in Argentinean yoghurts during refrigerated storage. Food Res. Int. 33:97-102.

Wang, J., Z. Guo, Q. Zhang, L. Yan, W. Chen, X. M. Liu, and H. P. Zhang. 2009. Fermentation characteristics and transit tolerance of probiotic Lactobacillus casei Zhang in soymilk and bovine milk during storage. J. Dairy Sci. 92:2468-2476.

Yu, J., Z. Sun, W. Liu, Q. Bao, J. Zhang, and H. Zhang. 2012. Phylogenetic study of Lactobacillus acidophilus group, L. casei group and L. plantarum group based on partial hsp60, pheS and tuf gene sequences. Eur. Food Res. Technol. 234:927-934.

Yu, J., W. H. Wang, B. L. G. Menghe, M. T. Jiri, H. M. Wang, W. J. Liu, Q. H. Bao, Q. Lu, J. C. Zhang, F. Wang, H. Y. Xu, T. S. Sun, and H. P. Zhang. 2011. Diversity of lactic acid bacteria associated with traditional fermented dairy products in Mongolia. J. Dairy Sci. 94:3229-3241.

Zhang, H. P., Y. F. Chen, W. J. Liu, Z. H. Sun, Q. H. Bao, and W. Y. Zhang. 2012. Biological diversity of lactic acid bacteria in natural fermented dairy products. 1st ed. Science Press, Beijing, China. 